\title{
Respiratory Therapist-Managed Arterial Catheter Insertion and Maintenance Program: Experience in a Non-Teaching Community Hospital
}

\author{
Sasheen Pack RRT, Peter Y Hahn MD MBA, James K Stoller MD MSc FAARC, and \\ Srinivas R Mummadi MD
}

\begin{abstract}
BACKGROUND: Usual practice in community health-care settings indicates that arterial catheters are inserted by physicians. In the context of a respiratory therapist (RT)-managed arterial catheter placement protocol being implemented in our community hospital, the current study describes the implementation and outcomes of this RT-managed arterial catheter insertion and maintenance program. METHODS: Tuality Healthcare is a 215-bed community health-care system (10-bed ICU) in Hillsboro, Oregon. With the goal of enhancing the quality of ICU care, an RT-managed multidisciplinary team was implemented to lead the delivery of protocolized ventilator liberation, arterial catheter insertion, and arterial blood gas utilization. Preparation for the program included didactic teaching, simulation-based training, and precepted procedural experience. A database was created for audit and quality improvement purposes. Outcomes and arterial blood gas utilization data were obtained from the audit database and from the hospital electronic health record. RESULTS: During the 4-y period (March 1, 2012, to April 31, 2016), 256 arterial catheter insertion attempts were made by a team of 12 qualified RTs. The success rate for the initial placement attempt by RT was high (94.5\% [242 of 256]). Sixty-three percent of arterial lines were placed in patients to help manage severe sepsis/septic shock. No ischemic or infectious complications were reported during the study period. Nearly $40 \%$ (96 of 242) of the successful placements by RTs on initial attempts were performed during the night shift, when intensivists were not physically present in the ICU. CONCLUSIONS: This experience establishes the feasibility of an RT-managed arterial catheter placement program in a community ICU. The RT-managed program was characterized by a high degree of success and safety and allowed arterial catheter placement at times when intensivists were not available in the ICU. This experience extends the sparse reported experience of RT-managed arterial catheter placement programs and underscores the value of RTs as members of the ICU team. Key words: respiratory therapist-managed arterial catheterization program; arterial catheterization; respiratory care protocols. [Respir Care 2017;62(12):1520-1524. (C) 2017 Daedalus Enterprises]
\end{abstract}

\section{Introduction}

Arterial catheters are a mainstay of ICU care in the United States, with utilization frequencies of $22-35 \%^{1}$

\footnotetext{
Ms Pack is affiliated with the Cardiopulmonary Department, Tuality Healthcare, Hillsboro, Oregon. Dr Hahn is the Chief Medical Officer of Metro Health-University of Michigan, Wyoming, Michigan and is affiliated with the Department of Pulmonary and Critical Care Medicine, Metro Health-University of Michigan Health, Wyoming, Michigan. Dr Mummadi is affiliated with the Department of Pulmonary and Critical Care Medicine, Metro Health-University of Michigan Health, Wyoming,
}

among the 4.14 million ICU admissions each year. ${ }^{2}$ Arterial catheters are used for directing hemodynamic management and for delivering protocolized care.

Usual practice in non-academic medical centers is for physicians to place arterial catheters in the ICU. In the context of widespread overall adoption of respiratory ther-

\footnotetext{
Michigan. Dr Stoller is affiliated with the Education Institute, Cleveland Clinic Lerner College of Medicine, Cleveland, Ohio.

Dr Hahn has disclosed a relationship with Medtronic Super Dimension. The other authors have disclosed no conflicts of interest.
} 


\section{RT-Managed Arterial Catheter Protocol}

apy protocols in academic ICUs in the United States, 2 reports describe experience with respiratory therapist (RT)managed arterial catheter programs, ${ }^{3,4}$ but none is available regarding experience from a community hospital ICU.

\section{See the Related Editorial on Page 1613}

To extend the reported favorable experience with RT protocols, the current report describes our experience with an RT-managed arterial catheter program in a community hospital in Oregon.

\section{Methods}

In the context of this observational study, an RT-managed arterial catheterization program (see the supplementary methods at http://www.rcjournal.com) was implemented at Tuality Healthcare, a 215-bed community hospital with a 10-bed ICU, on March 1, 2012. In this program, RTs were responsible for initial evaluation for arterial catheter insertion, set-up of a blood-conserving pressure line, arterial catheter insertion, and daily line maintenance. The current study reports the observed experience with this program for the 4-y interval between March 1, 2012, and April 31, 2016.

Tuality Healthcare's ICU incorporated a Leapfrog-compliant high-intensity model ${ }^{5}$ in November 2011 , which included as key features: intensivist co-management with admitting hospitalists, daily multidisciplinary rounds (ICU doctor, pharmacist, registered nurse, RT, physical therapist, case management registered nurse, and an infection control practitioner), and ICU directorship by an intensivist.

Intensivists were present in the ICU during daytime hours and were available through a telemedicine-enabled intermediary at night. Admissions and nighttime crosscoverage were delivered by admitting hospital medicine practitioners. A total of 2 pulmonary/critical care practitioners comprised the critical care group during the study period. During the night, emergent critical care interventions, such as insertion of endotracheal tube and central venous catheters, were performed by in-house emergency medicine physicians and anesthesiologists. Before the introduction of the RT-managed arterial catheter program, the birth unit's mid-level anesthesia practitioner was consulted if an arterial catheter was deemed necessary during

\footnotetext{
Correspondence: Srinivas R Mummadi MD, Metro Health-University of Michigan Health, 2122 Health Drive SW, Suite 220, Wyoming, MI 49519. E-mail: Srinivas.Mummadi@metrogr.org.
}

Supplementary material related to this paper is available at http://www.rcjournal.com.

DOI: $10.4187 /$ respcare. 05486

\section{QUICK LOOK}

\section{Current knowledge}

Respiratory therapist (RT)-led protocolized care has been shown to be effective. The safety of RT-managed arterial catheter insertion and maintenance protocols has been sparsely described only in academic and large community health-care systems.

\section{What this paper contributes to our knowledge}

An RT-managed arterial catheter program can be established in a small community hospital ICU with a high degree of technical success and safety. The availability of RTs to place arterial catheters at times when physicians were unavailable in this setting suggests that such programs can also enhance the timeliness of ICU care.

the night. As part of an ICU quality initiative, several programs were implemented in 2012, namely a ventilator liberation protocol and an RT-managed arterial catheterization program, which also allocated decisions regarding drawing arterial blood gases to RTs.

Careful planning for the program was required because none of the RTs had prior experience in placing arterial catheters. Criteria for RT inclusion included having $\geq 5 \mathrm{y}$ of critical care experience and a willingness to engage in the training to participate.

Pre-implementation activities included didactic teaching sessions, simulation-based training programs, and precepted experience. RTs were trained to use the Arrow 20Ga catheterization kit (REF RA-04020, Teleflex, Morrisville, North Carolina) and the handheld dopplex D900 vascular Doppler probe (ArjoHuntleigh, Addision, Illinois).

During the pre-implementation didactic phase, RTs were educated regarding the indications for arterial catheter insertion, device set-up, contraindications, complications, proper placement technique, and arterial catheter troubleshooting. Training included implementation of infection prevention bundles comprising hand hygiene, aseptic technique, maximal sterile barrier precautions during insertion, optimal catheter site selection, and catheter site dressing regimens. After successful completion of the competency checklist and 5 successful precepted insertions, the RT was allowed to place arterial catheters without a preceptor. A comprehensive module including the learning objectives, teaching materials, a post-test, and a checklist for demonstrating acquired competency are presented as online (see supplementary methods).

A database (Excel, Microsoft, Redmond, Washington) was created for audit and quality improvement purposes. Data fields were defined a priori and included: the name of the RT performing the insertion, the date of attempted 


\section{RT-Managed Arterial Catheter Protocol}

cannulation, use of Doppler guidance, diagnostic indications for arterial catheter placement, and all complications (including catheter-related complications) recorded during the ICU stay. In keeping with widespread practice for implementing RT protocol programs, a physician order requesting RT placement of an arterial catheter was required. After the arterial catheter was placed, the protocol mandated use of a closed blood draw system ${ }^{6,7}$ and daily monitoring by the ICU doctor/registered nurse/infection control practitioner team to ensure functionality and to assess for any signs of infection. The Centers for Disease Control definition of catheter-related bloodstream infection $^{8}$ was used, and all such instances were reported to the National Healthcare Safety Network database by the institution's infection preventionist. The protocol also mandated daily discussions during multidisciplinary rounds about the continued need for an arterial catheter. All complications related to arterial catheters (eg, pseudoaneurysms, septic thromboarteritis, and bleeding after removal) were assessed by the rounding intensivist and recorded during the multidisciplinary rounds for entry into the study database.

After an initial 4-member team was formed, the program was officially implemented on March 1, 2012. Postimplementation activities included an experiential model of learning, annual competency renewal, and incorporation of direct feedback from physicians and nursing staff on the proceduralists' practice.

Arterial blood gas volumes during each fiscal year and data about annual ICU admissions were extracted from the hospital electronic health record (Cerner, Kansas City, Missouri). Data regarding average daily ICU census were obtained from the hospital's Department of Finance comparative statistical report. This descriptive study of an implemented ICU practice was reviewed by the Western Institutional Review Board (Puyallup, Washington) (submission 1-991652-1) and was deemed exempt from the need for individual patient consent.

\section{Results}

During the study period (March 1, 2012, to April 31, 2016), 12 of 16 total RTs on staff (75\%) successfully completed the credentialing process. A total of 256 arterial catheter insertion attempts were undertaken, and successful placement on the initial placement attempt was documented in 242 instances (94.5\%; 226 radial, 15 brachial, and 1 dorsalis pedis placement locations). Arterial catheter placements universally employed a handheld Doppler probe (dopplex D900 vascular Doppler probe) and indications for catheter placement are described in Table 1. Indications grouped in the "Others" category include cardiogenic shock $(n=11,4.2 \%)$, hypertensive urgency $(n=6,2.3 \%)$, anaphylactic shock $(n=6,2.3 \%)$, and drug overdose
Table 1. Indications for Arterial Catheter Placement Grouped by Primary Diagnosis: 2012-2016

\begin{tabular}{lc}
\hline \multicolumn{1}{c}{ Primary Diagnosis } & Number Performed* \\
\hline Severe sepsis/septic shock & 158 \\
Acute respiratory failure/ARDS & 30 \\
Sudden cardiac death & 35 \\
Others & 27 \\
& \\
$*$ There were 242 insertions by RTs on initial attempt, and 8 insertions by RTs and others \\
following an initial unsuccessful attempt, for a total of 250 insertions. We excluded 3 \\
unsuccessful attempts and 3 instances where further attempts were not pursued. \\
\hline
\end{tabular}

$(n=4,1.5 \%)$. Notably, $40 \%$ (96 of 242) of the successful placements on initial attempts were performed during the night shift, when intensivist physicians were not physically present in the ICU.

Of the 14 unsuccessful initial placement attempts, in 4 instances, the $\mathrm{AC}$ was ultimately successfully placed by a different RT; in 3 instances, a femoral AC was placed by an intensivist physician; in 3 cases, intensivists were also unable to place an $\mathrm{AC}$; in 1 instance, a radial $\mathrm{AC}$ was placed by a cardiac surgery registered nurse; and in 3 instances, no further attempts to place an $\mathrm{AC}$ were undertaken.

No ischemic complications or further diagnostic studies (ie, MRI, ultrasound, angiogram) or interventions (ie, heparin, thrombolytics, surgery) were reported. A case of inability to achieve hemostasis with digital pressure after the removal of radial artery catheter was recorded. A total of 7 bloodstream infections related to arterial catheter use were recorded over the $4 \mathrm{y}$ of the study ( 2 in 2011, none in 2012-2014, 3 in 2015, and 2 in 2016). In no case of a bloodstream infection was the arterial catheter in place for $>2$ calendar days before the event.

The annual number of arterial blood gas analyses performed in the ICU steadily declined (Fig. 1) over the study interval (from 874 in 2011 to 319 in 2015), whereas the number of annual ICU admissions was largely unchanged. Average daily ICU census ranged from 3 to 4 over the study interval.

\section{Discussion}

In keeping with prior experience, which was limited to a teaching hospital and a large community hospital, $, 3,4$ this report shows that an RT-managed arterial catheter insertion and maintenance program can be successfully and safely implemented over $4 \mathrm{y}$ in a community mixed ICU.

The study findings add to the substantial experience in which RTs can assume expanded roles in the care of ICU patients $^{9-13}$ and can contribute to enhanced quality and patient safety, which is a major current focus in health care. In 2001, the Institute of Medicine provided an ana- 


\section{RT-Managed Arterial Catheter Protocol}

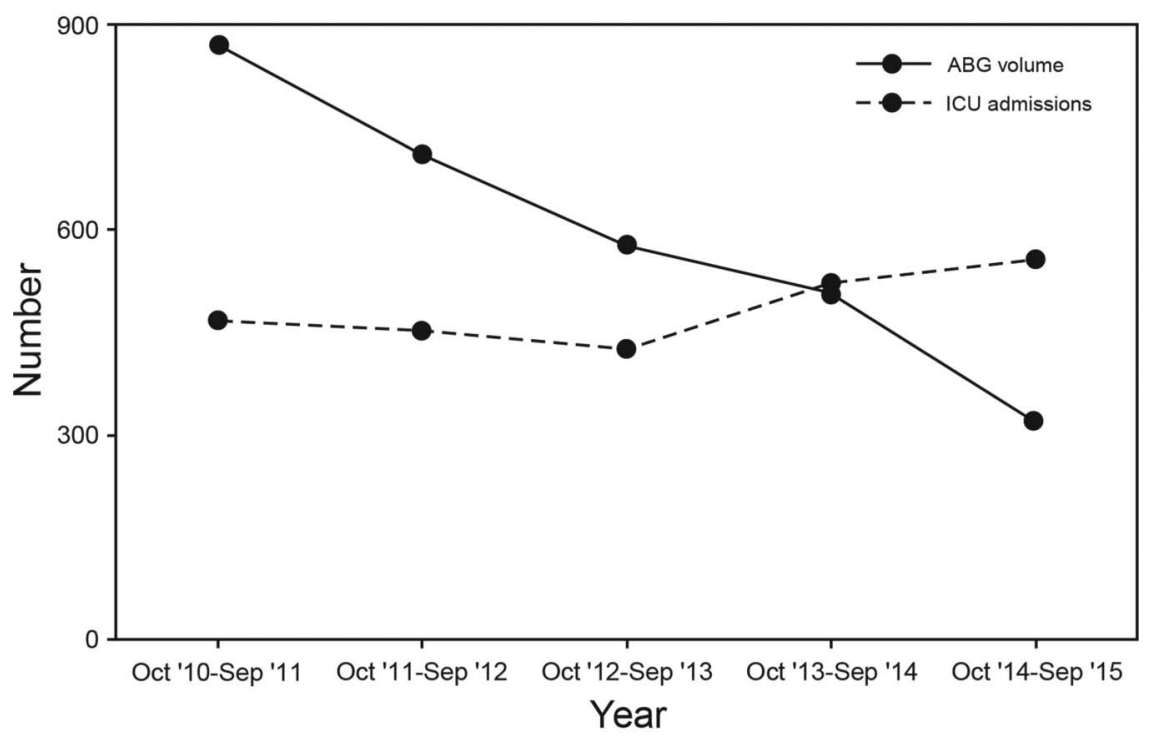

Fig. 1. Trends in arterial blood gas (ABG) utilization compared with ICU admissions (2010-2015).

lytical framework of health-care quality based on 6 domains: patient-centered, timely, efficient, effective, equitable, and safe care. ${ }^{14}$ This experience aligns with the Institute of Medicine report by demonstrating that RTs can place arterial catheters safely and in a timely way. For example, $40 \%$ of the arterial catheters placed in this series were placed during the night, which was facilitated by the 24/7 presence of RTs in the ICU. Were it not for the RT availability during the night, placement of these arterial catheters might have been delayed.

In demonstrating that a task such as arterial catheter placement (which has traditionally been delegated only to physicians) can be safely assumed by RTs, this experience also adds to the expanded scope of practice that allied care practitioners can take on (physician, RT, ${ }^{13}$ nurse practitioner, ${ }^{15}$ and physician assistant ${ }^{16}$ teams), thereby contributing to top-of-license initiatives. That RTs are functioning at the top of their license in placing arterial catheters also contributes to optimizing the efficiency of intensivists and may favorably affect physician burnout. ${ }^{17}$ Experts have used complexity science ${ }^{18,19}$ to better understand workflow, ${ }^{20}$ collaborative work, ${ }^{20}$ and patient safety in the ICU. ${ }^{21}$ In this context, an RT, and especially one who is functioning at top-of-license, is an important contributor to the diversity and robustness of a complex adaptive system, such as the ICU.

Although insertion of arterial catheters is a common practice in the ICU, experts have raised concerns about their effectiveness. ${ }^{22-24}$ Consequences related to overutilization, such as anemia of chronic investigation ${ }^{25,26}$ and promotion of overtesting ${ }^{7,23}$ (arterial blood gas analysis costs) with no attendant improvement in outcomes such as survival ${ }^{24}$ help inform the debate regarding the value of placing arterial catheters. At the same time, arterial cath- eters continue to be important in helping guide resuscitation of shock in most instances; for example, $62 \%$ of subjects received arterial catheter-directed resuscitation in the usual care arm of the ProMISe trial. ${ }^{27}$ Although definite attribution is difficult, it is noteworthy that the number of arterial blood gas analyses ordered in our ICU decreased over the time that the RT-led insertion program was implemented. We speculate that this reduction may reflect an impact of the program. For example, much attention was given to achieving interdisciplinary consensus about indications for arterial catheter use before the program was launched and incorporating this program as part of a wider ICU quality improvement project based on protocolizing care. Co-interventions (ventilator liberation order sets utilizing noninvasive assessment of oxygenation and ventilation) probably also contributed to the observed reduction in arterial blood gas analysis utilization. ${ }^{28}$

This study has limitations. First, the data are observational without concurrent or historical controls. Data regarding outcomes of the few physician-placed arterial catheters during the 4-y study interval (eg, in the operating room or cardiac catheterization laboratory) were unavailable, precluding comparison of RT with physician performance. Still, the high rate of successful initial placement and the low rate of complications with RT-placed arterial catheters in this study reinforce the success of the program and advance proof-of-concept that such programs can be effective in small, community-based ICUs. As a second limitation, some process measures regarding catheter use were unavailable (eg, the amount of time an arterial catheter was left in the vessel, the volume of blood drawn, and the incidence of new anemia over the course of ICU stay). Third, ideally, an analysis of arterial catheter utilization would compare length of ICU stay rather than ICU admis- 


\section{RT-Managed Arterial Catheter Protocol}

sions to fully consider the time over which arterial catheter placement was possible. Data on length of stay in the ICU could not be extracted from the hospital's electronic medical record.

\section{Conclusions}

In summary, our study shows that an arterial catheter insertion and maintenance program managed by RTs can be implemented in a non-teaching community hospital ICU and that the program can deliver effective and safe care. This RT-managed program facilitated placement of arterial catheters at times when physician intensivists were not available, thereby potentially enhancing the timeliness of care as well. This experience establishes the feasibility of establishing an RT-managed arterial catheter program in a small community hospital ICU and extends the sparse reported experience with such programs.

\section{REFERENCES}

1. Gershengorn HB, Garland A, Kramer A, Scales DC, Rubenfeld G, Wunsch $\mathrm{H}$. Variation of arterial and central venous catheter use in United States intensive care units. Anesthesiology 2014;120(3):650664.

2. Mullins PM, Goyal M, Pines JM. National growth in intensive care unit admissions from emergency departments in the United States from 2002 to 2009. Acad Emerg Med 2013;20(5):479-486.

3. Gronbeck C, 3rd, Miller EL. Nonphysician placement of arterial catheters: experience with 500 insertions. Chest 1993;104(6):17161717.

4. Rowley DD, Mayo DF, Durbin CG Jr. Initial experience with a respiratory therapist arterial line placement service. Respir Care 2000; 45(5):482-485.

5. Logani S, Green A, Gasperino J. Benefits of high-intensity intensive care unit physician staffing under the Affordable Care Act. Crit Care Res Pract 2011;2011:170814.

6. Silver MJ, Jubran H, Stein S, McSweeney T, Jubran F. Evaluation of a new blood-conserving arterial line system for patients in intensive care units. Crit Care Med 1993;21(4):507-511.

7. Silver MJ, Li YH, Gragg LA, Jubran F, Stoller JK. Reduction of blood loss from diagnostic sampling in critically ill patients using a blood-conserving arterial line system. Chest 1993;104(6):1711-1715.

8. Tomlinson D, Mermel LA, Ethier MC, Matlow A, Gillmeister B, Sung L. Defining bloodstream infections related to central venous catheters in patients with cancer: a systematic review. Clin Infect Dis 2011;53(7):697-710.

9. Stoller JK. The rationale for therapist-driven protocols. Respir Care Clin N Am 1996;2(1):1-14.

10. Stoller JK. The effectiveness of respiratory care protocols. Respir Care 2004;49(7):761-765.

11. Stoller JK, Mascha EJ, Kester L, Haney D. Randomized controlled trial of physician-directed versus respiratory therapy consult service- directed respiratory care to adult non-ICU inpatients. Am J Respir Crit Care Med 1998;158(4):1068-1075.

12. Ely EW, Meade MO, Haponik EF, Kollef MH, Cook DJ, Guyatt GH, Stoller JK. Mechanical ventilator weaning protocols driven by nonphysician health-care professionals: evidence-based clinical practice guidelines. Chest 2001;120(6 Suppl):454S-463S.

13. Modrykamien AM, Stoller JK. The scientific basis for protocoldirected respiratory care. Respir Care 2013;58(10):1662-1668.

14. Institute of Medicine Committee on Quality of Health Care in America. Crossing the quality chasm: a new health system for the 21 st century. Washington, DC: National Academy Press; 2001. http:// www.nap.edu/books/0309072808/html/. Accessed September 1, 2017.

15. Landsperger JS, Semler MW, Wang L, Byrne DW, Wheeler AP. Outcomes of nurse practitioner-delivered critical care: a prospective cohort study. Chest 2016;149(5):1146-1154.

16. Costa DK, Wallace DJ, Barnato AE, Kahn JM. Nurse practitioner/ physician assistant staffing and critical care mortality. Chest 2014; 146(6): 1566-1573.

17. Howell MD. Intensivist time allocation: economic and ethical issues surrounding how intensivists use their time. Semin Respir Crit Care Med 2012;33(4):401-412.

18. Plsek PE, Greenhalgh T. Complexity science: the challenge of complexity in health care. BMJ 2001;323(7313):625-628.

19. Rouse WB. Health care as a complex adaptive system: implications for design and management. Bridge Washington-National Academy of Engineering 2008;38(1):17.

20. Kannampallil TG, Schauer GF, Cohen T, Patel VL. Considering complexity in healthcare systems. J Biomed Inform 2011;44(6):943947.

21. Dong Y, Chbat NW, Gupta A, Hadzikadic M, Gajic O. Systems modeling and simulation applications for critical care medicine. Ann Intensive Care 2012;2(1):18.

22. Garland A. Arterial lines in the ICU: a call for rigorous controlled trials. Chest 2014;146(5):1155-1158.

23. Garland A, Connors AF Jr. Indwelling arterial catheters in the intensive care unit: necessary and beneficial, or a harmful crutch? Am J Respir Crit Care Med 2010;182(2):133-134.

24. Gershengorn HB, Wunsch H, Scales DC, Zarychanski R, Rubenfeld G, Garland A. Association between arterial catheter use and hospital mortality in intensive care units. JAMA Intern Med 2014;174(11): 1746-1754.

25. Hayden SJ, Albert TJ, Watkins TR, Swenson ER. Anemia in critical illness: insights into etiology, consequences, and management. Am J Respir Crit Care Med 2012;185(10):1049-1057.

26. Smoller BR, Kruskall MS. Phlebotomy for diagnostic laboratory tests in adults: pattern of use and effect on transfusion requirements. N Engl J Med 1986;314(19):1233-1235.

27. Mouncey PR, Osborn TM, Power GS, Harrison DA, Sadique MZ, Grieve RD, et al. Trial of early, goal-directed resuscitation for septic shock. N Engl J Med 2015;372(14):1301-1311.

28. Pilon CS, Leathley M, London R, McLean S, Phang PT, Priestley R, et al. Practice guideline for arterial blood gas measurement in the intensive care unit decreases numbers and increases appropriateness of tests. Crit Care Med 1997;25(8):1308-1313.

This article is approved for Continuing Respiratory Care Education credit. For information and to obtain your CRCE

(free to AARC members) visit

www.rcjournal.com

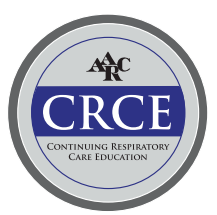

